\title{
Comparative Fluorescence Resonance Energy Transfer Study in Pluronic Triblock Copolymer Micelle and Niosome Composed of Biological Component Cholesterol : An Investigation of Effect of Cholesterol and Sucrose on the FRET parameters
}

\author{
Arpita Roy, Niloy Kundu, Debasis Banik, and Nilmoni Sarkar*
}

Department of Chemistry, Indian Institute of Technology, Kharagpur 721302, WB, India E-mail: nilmoni@chem.iitkgp.ernet.in

Fax: 91-3222-255303

\section{Instrumentations:}

\subsection{Malvern Nano ZS instrument:}

Dynamic light scattering (DLS) measurements have been performed using Malvern Nano ZS instrument. In this instrument, a $4 \mathrm{~mW} \mathrm{He}-\mathrm{Ne}$ laser $(\lambda=632 \mathrm{~nm})$ is used and detector is poisoned at $173^{\circ}$ angle. The hydrodynamic diameter $\left(\mathrm{d}_{\mathrm{h}}\right)$ of particles was calculated by utilizing the collected scattering intensity according to following equation:

$$
d_{h}=\frac{k_{B} T}{3 \pi \eta D}
$$

Where, $\mathrm{k}_{\mathrm{B}}, \mathrm{T}, \mathrm{D}$ and $\eta$ denotes Boltzmann constant, Temperature, diffusion coefficient and viscosity respectively.

\subsection{Transmission Electron Microscopy Measurements:}

Transmission electron microscopy (TEM) experiments using was carried out the JEOL model JEM 2100 transmission electron microscope at an operating voltage of $200 \mathrm{kV}$ for the structural characterization of niosomes in absence and presence of sucrose. TEM images of the niosomes were taken by using $0.5 \mathrm{wt} \%$ of uranyl acetate as the staining agent.

\subsection{Fluorescence Correlation Spectroscopy (FCS) Measurement:}

The three dimensional (3D) diffusion models have been utilized to fit the FCS traces. For K fraction of dye diffusing within a system with distinct diffusion constants, the correlation function $\mathrm{G}(\tau)$ is defined as, ${ }^{1}$ 


$$
G(\tau)=\frac{1}{N} \sum_{i=1}^{K} \varphi_{i}\left(1+\frac{\tau}{\tau_{i}}\right)^{-1}\left(1+\frac{\tau}{\tau_{i} S^{2}}\right)^{-0.5}
$$

Here, $\mathrm{N}$ denote the number of fluorescent species with the focal volume, $\varphi_{i}$ is the fractional weighting factor for the $\mathrm{i}^{\text {th }}$ contribution to the autocorrelation curve and $\tau_{\mathrm{i}}$ is the diffusion time of the fluorescent species within the observation volume and $\tau$ is the delay or lag time. $\mathrm{S}$ denotes the structure parameter of the excitation volume. The structural parameter $(\mathrm{S})$ is defined as $(1 / \mathrm{r})$, where 1 is the longitudinal radii and $r$ is the transverse radii. Transverse radii ( $r$ ) can be determined through the fitting of an autocorrelation curve of a fluorescent species with known diffusion constant. We have used R6G in water for this purpose and the diffusion coefficient $\left(D_{t}\right)$ is $426 \mu \mathrm{m}^{2} \mathrm{~s}^{-1}{ }^{2}$. The curves are fitted with the following equation in order to determine the global parameter $\mathrm{r}$ and $\mathrm{S}$.

$$
G(\tau)=\frac{1}{N}\left(1+\frac{4 D_{t} \tau}{r^{2}}\right)^{-1}\left(1+\frac{4 D_{t} \tau}{S^{2} r^{2}}\right)^{-0.5}
$$

Where $D_{t}$ the diffusion coefficient of the fluorescent species and $\tau$ is the diffusion time and in the fitting analysis $\mathrm{r}$ and $\mathrm{S}$ are kept as linked global parameter. $\mathrm{S}=5$ is obtained after fitting the correlation curves of R6G in water and observation volume $\left(\mathrm{V}_{\mathrm{eff}}\right)$ is obtained from the following equation,

$$
V_{e f f}=\pi^{3 / 2} r^{3} S
$$

The final value of $\mathrm{r}$ is obtained as $365 \mathrm{~nm}$ and $\mathrm{V}_{\text {eff }}$ is $1.35 \mathrm{fl}$. All the FCS experiments were performed at $25^{\circ} \mathrm{C}$ and the diffusion coefficient can be obtained from the following equation,

$$
D_{t}=\frac{r^{2}}{4 \tau}
$$


Table S1: The Proportion of the Membrane Components and Dyes (Donor and Acceptor)

\begin{tabular}{|c|c|c|}
\hline $\begin{array}{l}\text { Systems } \\
\end{array}$ & Proportion & \\
\hline & {$[$ Donor $] /[$ Cholesterol $]$} & $1.27 \times 10^{-3}$ \\
\hline F127-Cholesterol & [Donor]/[F127] & $0.64 \times 10^{-3}$ \\
\hline \multirow[t]{2}{*}{ Niosome at $R=0.5$} & [Acceptor]/[Cholesterol] & $4.36 \times 10^{-3}$ \\
\hline & [Acceptor]/[F127] & $2.18 \times 10^{-3}$ \\
\hline \multirow{4}{*}{$\begin{array}{l}\text { F127-Cholesterol } \\
\text { Niosome at } R=1.0\end{array}$} & [Donor]/[Cholesterol] & $0.64 \times 10^{-3}$ \\
\hline & [Donor]/ [F127] & $0.64 \times 10^{-3}$ \\
\hline & [Acceptor]/[Cholesterol] & $2.18 \times 10^{-3}$ \\
\hline & [Acceptor]/[F127] & $2.18 \times 10^{-3}$ \\
\hline
\end{tabular}

Table S2: Time-Resolved Fluorescence Decay Parameters of C153 Different Excitation Wavelengths

\begin{tabular}{lllllll}
\hline System & $\begin{array}{l}\lambda_{\text {ex }} \\
(\mathbf{n m})\end{array}$ & \multicolumn{1}{c}{$\boldsymbol{\alpha}_{\mathbf{1}}$} & $\boldsymbol{\alpha}_{\mathbf{2}}$ & $\boldsymbol{\tau}_{\mathbf{1}}(\mathbf{n s})$ & $\boldsymbol{\tau}_{\mathbf{2}}(\mathbf{n s})$ & $\begin{array}{l}\left\langle\boldsymbol{\tau}_{\text {avg }}>^{\mathbf{a}}\right. \\
(\mathbf{n s})\end{array}$ \\
\hline F127 micelle & 408 & 0.21 & 0.79 & 2.89 & 5.72 & 5.12 \\
& 440 & 0.30 & 0.70 & 2.85 & 5.66 & 4.82 \\
\hline F127 Cholesterol & 408 & 0.17 & 0.83 & 2.94 & 5.84 & 5.35 \\
Niosome at R=0.5 & 440 & 0.31 & 0.69 & 3.81 & 5.75 & 5.15 \\
\hline F127-Cholesterol & 408 & 0.13 & 0.87 & 3.00 & 6.01 & 5.62 \\
Niosome at R=1.0 & 440 & 0.32 & 0.68 & 4.46 & 5.84 & 5.40 \\
\hline R=1.0 in presence & 408 & 0.09 & 0.91 & 3.06 & 6.06 & 5.79 \\
of 1.3 (M) sucrose & 440 & 0.33 & 0.67 & 4.89 & 5.93 & 5.59 \\
\hline
\end{tabular}

\footnotetext{
${ }^{\mathrm{a}}$ Experimental error of $\pm 5 \%$.
} 
Table S3: Diffusion coefficients $\left(D_{t}\right)$ of R6G in water, micelle and cholesterol containing niosome at $\mathrm{R}=1.0$

\begin{tabular}{cc}
\hline System & $\boldsymbol{D}_{\boldsymbol{t}}\left(\boldsymbol{\mu \mathbf { m } ^ { 2 } \mathbf { S } ^ { - 1 } ) ^ { \mathbf { a } }}\right.$ \\
\hline R6G in Water & $426(100 \%)$ \\
\hline R6G in F127 micelle & $69(88 \%)$ \\
\hline R6G in F127/Cholesterol & $45(91 \%)$ \\
Niosome at R=1.0 & \\
\hline rimental error of $\pm 3 \%$ &
\end{tabular}

\section{${ }^{a}$ Experimental error of $\pm 3 \%$}
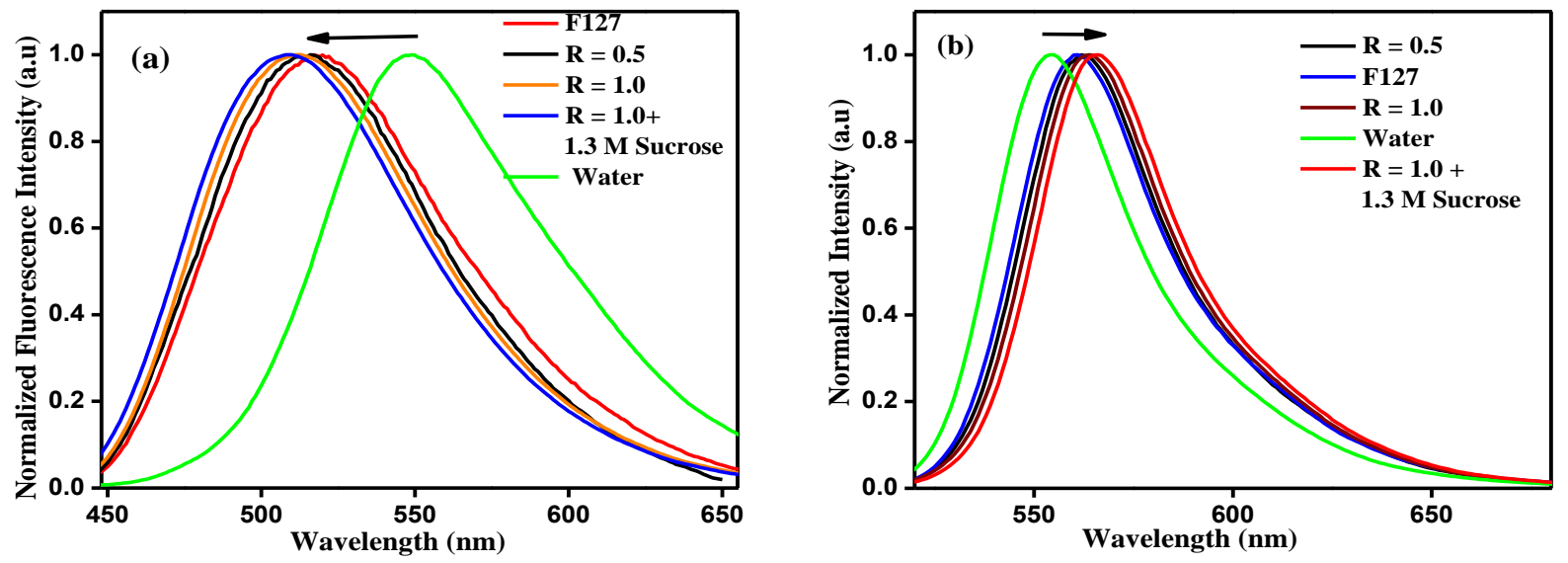

Figure S1: Normalised steady-state fluorescence spectra of (a) C153 and (b) R6G in F127 micelle, niosome containing Cholesterol/F127 at $\mathrm{R}=0.5, \mathrm{R}=1.0$ in presence and absence of Sucrose and also in water. 

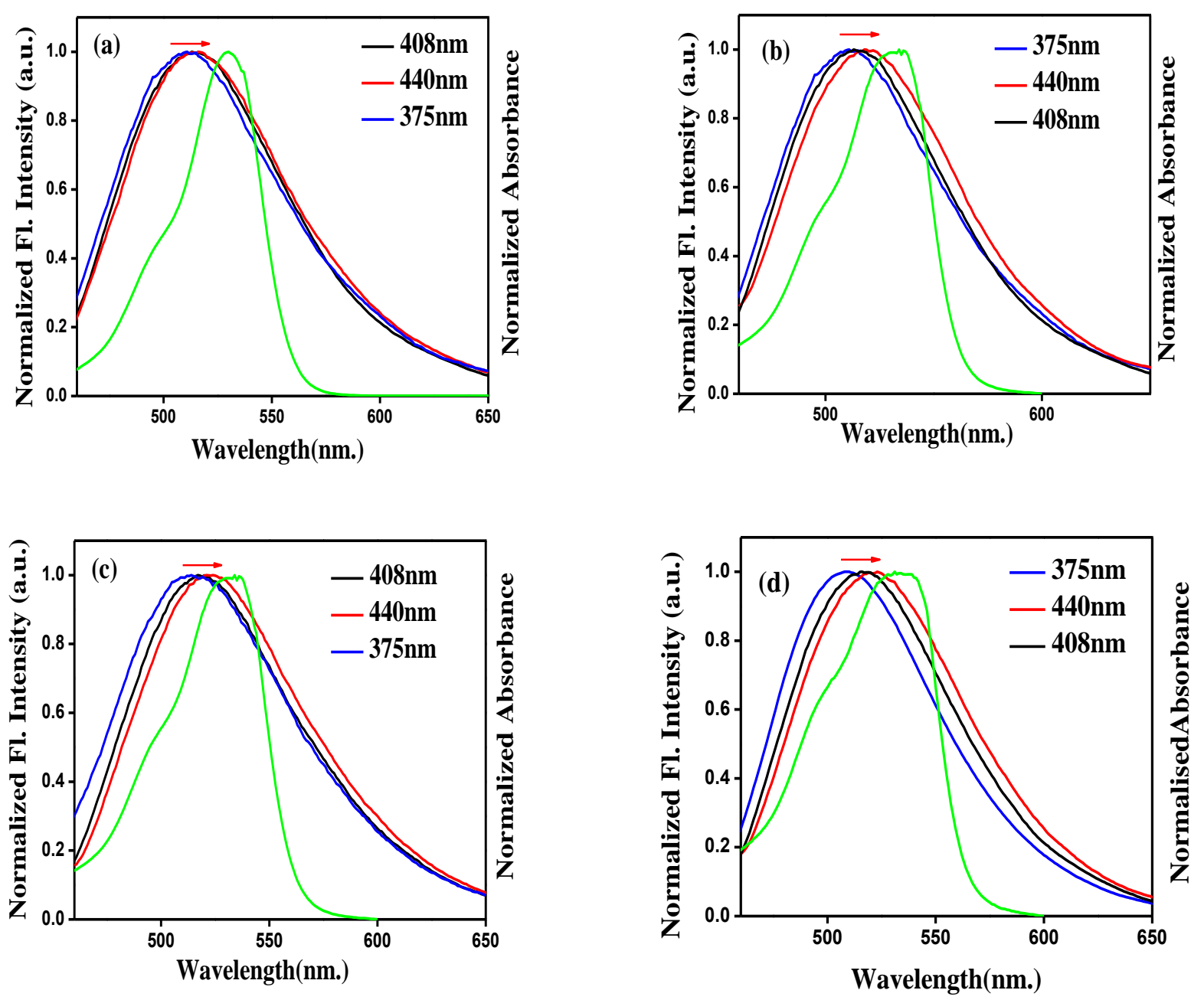

Figure S2: Spectral overlap between the steady-state fluorescence emission spectra of C153 with the absorption spectra of R6G in (a) F127 micelle, (b) Niosome containing F127 / Cholesterol at $\mathrm{R}=0.5$, (c) Niosome containing F127/ Cholesterol at $\mathrm{R}=1.0$ and (d) Niosome in presence of sucrose. 

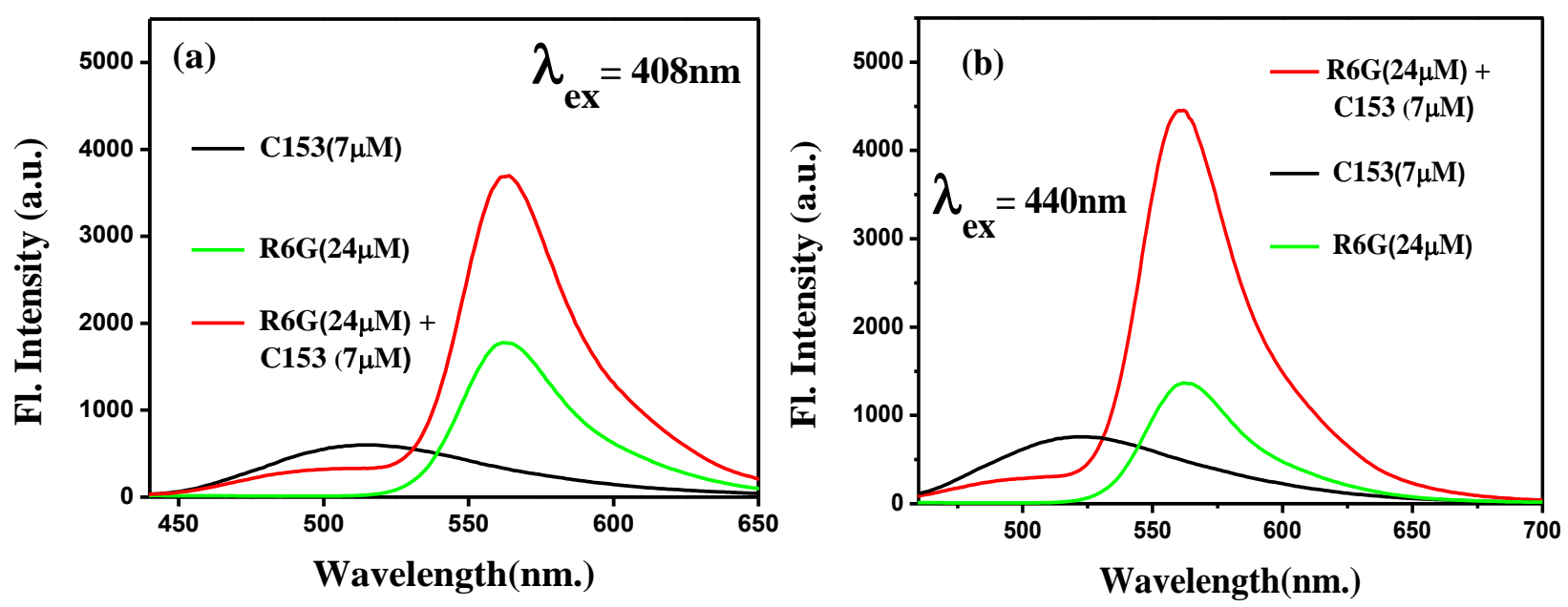

Figure S3: Fluorescence emission spectra of $\mathrm{C} 153(7 \mu \mathrm{M})$ in the absence and presence of R6G $(24 \mu \mathrm{M})$ in different microenvironments of Niosome containing F127/ Cholesterol at $\mathrm{R}=0.5$ (a), (b) at different excitation wavelength
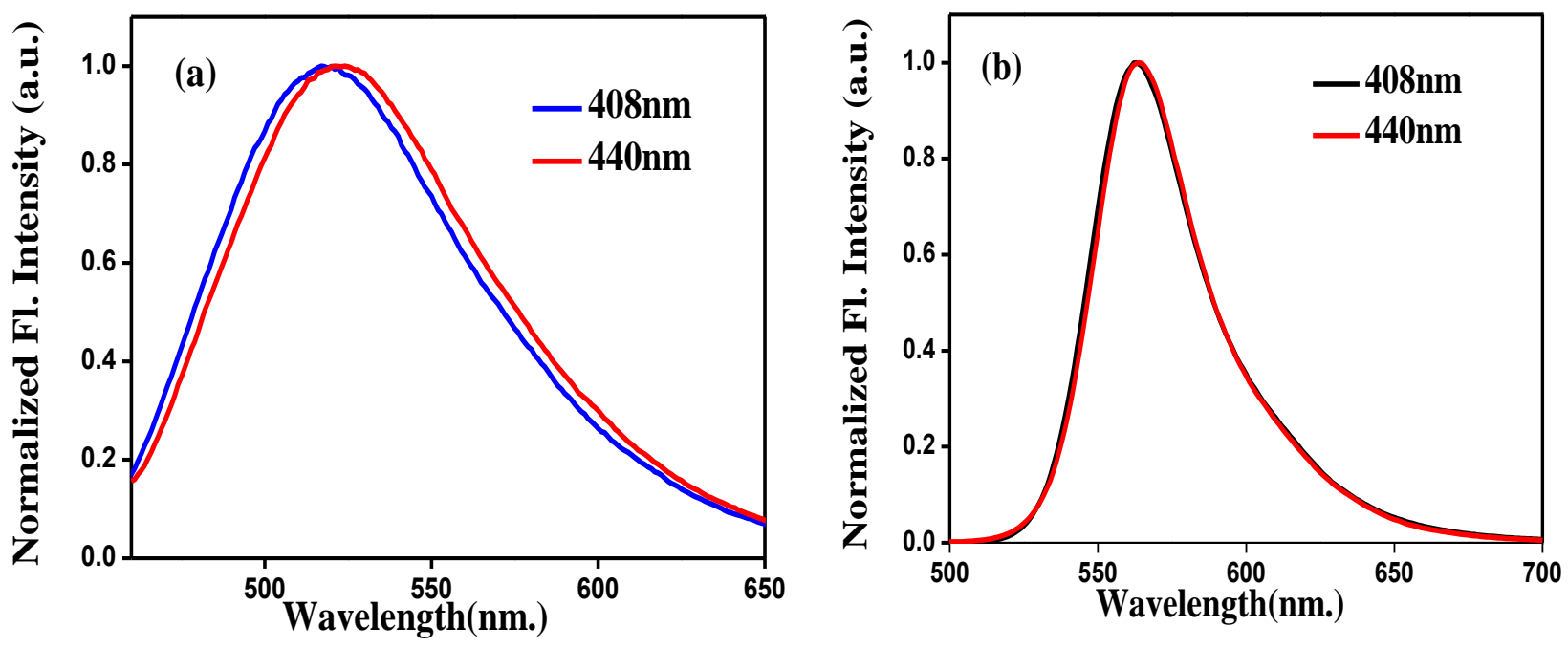

Figure S4: Normalised steady-state fluorescence spectra of (a) C153 and (b) R6G in Niosome containing F127 / Cholesterol at R=1.0 at different excitation wavelengths (408 and $440 \mathrm{~nm}$.) 

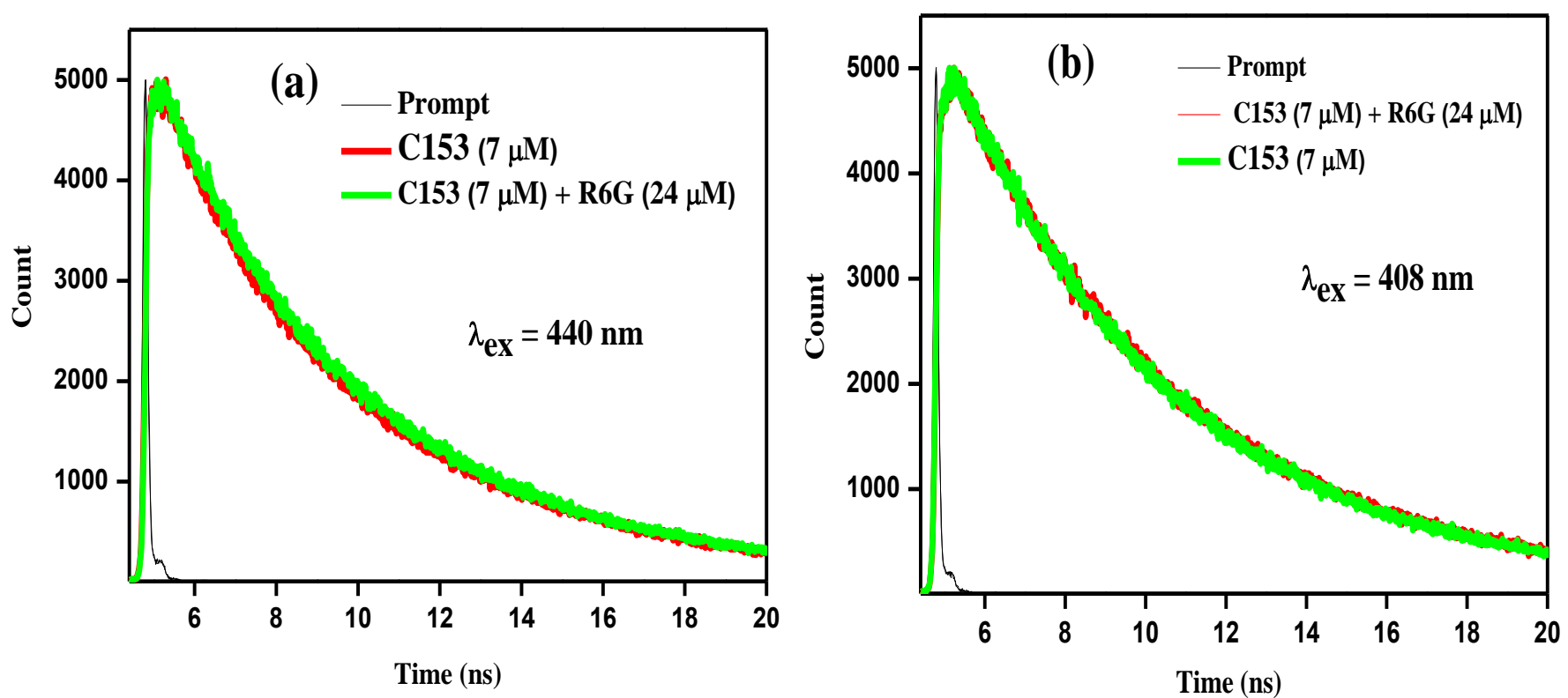

Figure S5: Time-resolved fluorescence decays of the donor $\mathrm{C} 153(7 \mu \mathrm{M})$ in the absence and presence of the acceptor R6G in Niosome containing [Cholesterol]/[F127] at R=1.0 (a), (b) at different excitation wavelength.

\section{References:}

(1) Lakowicz, J. R. Principles of Fluorescence Spectroscopy, 3rd ed.; Springer: New York, 2006.

(2) Petrasek, Z.; Schwille, P. Precise Measurement of Diffusion Coefficients using Scanning Fluorescence Correlation Spectroscopy. Biophys. J. 2008, 94, 1437-1448. 